\title{
MiR-146a expression profiles in osteoarthritis in different tissue sources: a meta-analysis of observational studies
}

\author{
Jia-Neng Liu, Song Lu and Chang-Ma Fu* (1)
}

\begin{abstract}
Background: MiR-146a has been widely studied in the pathogenesis of osteoarthritis (OA); however, the results are still controversial.

Objective: This meta-analysis analyzes the expression profile of miR-146a in various tissues of OA patients.

Methods: Public databases were searched for appropriate studies published up to September 1, 2021. A case-control study comparing the OA population and a non-OA healthy population was included.

Results: 26 articles were included in analysis. The results showed that the expression level of miR-146a in peripheral blood mononuclear cells (PBMCs) was significantly higher in OA patients than in controls (SMD: 1.23; $95 \% \mathrm{Cl}$ $0.08-2.37 ; p=0.035$ ) but not in plasma (SMD: $1.09 ; 95 \% \mathrm{Cl}-0.06,2.24 ; p=0.064)$. The expression level of miR-146a in cartilage was also significantly higher in OA patients than in controls (SMD: $6.39 ; 95 \% \mathrm{Cl} 0.36,12.4 ; p=0.038$ ) but not in chondrocytes (SMD: $-0.71 ; 95 \% \mathrm{Cl}-4.15,2.73 ; p=0.687$ ). The miR-146a level was significantly lower in synoviocytes in the OA population than in control patients (SMD: $-0.97 ; 95 \% \mathrm{Cl}-1.68,-0.26 ; p=0.008$ ). In synovial tissue, synovial fluid, and regulatory $T$ cells, there was no significant difference.
\end{abstract}

Conclusion: The expression level of miR-146a in cartilage tissue and PBMCs was significantly higher in OA patients than in non-OA healthy controls. Due to the limitations of this study, more research is needed to confirm these results in the future.

Trial registration: retrospectively registered.

Keywords: Osteoarthritis, MIR146A, MicroRNA, Diagnosis, Meta-analysis

\section{Background}

Osteoarthritis (OA) is a type of degenerative joint disease with a high incidence in the elderly population and is also the most common cause of activity restriction in adults. OA affects $7 \%$ of the world's population, while it is estimated that more than 240 million people suffer from symptomatic activity-restricted OA [1]. Population aging and an increasing proportion of obese people may lead

*Correspondence: fcm830_hfsy@163.com

Department of Orthopedics, The Third People's Hospital of Hefei, No. 204,

Wangjiang East Road, Baohe District, Hefei 230041, Anhui, China to a further increase in OA morbidity [2]. The diagnosis of OA mostly depends on clinical symptoms, and there is still a lack of specific biomarkers at present.

MicroRNAs are noncoding RNAs that are highly conserved and tissue specific and can inhibit gene translation into proteins and promote mRNA degradation to achieve posttranscriptional regulation. The complex interactions between microRNAs and their target genes play an important role in the pathogenesis and development of OA [3]. Of them, miR-146a has been widely studied for the pathogenesis of OA. Basic research suggests that miR-146a promotes chondrocyte apoptosis, cartilage original author(s) and the source, provide a link to the Creative Commons licence, and indicate if changes were made. The images or other third party material in this article are included in the article's Creative Commons licence, unless indicated otherwise in a credit line to the material. If material is not included in the article's Creative Commons licence and your intended use is not permitted by statutory regulation or exceeds the permitted use, you will need to obtain permission directly from the copyright holder. To view a copy of this licence, visit http://creativecommons.org/licenses/by/4.0/. The Creative Commons Public Domain Dedication waiver (http://creativeco mmons.org/publicdomain/zero/1.0/) applies to the data made available in this article, unless otherwise stated in a credit line to the data. 
damage, inflammation, and neovascularization $[4,5]$. It also suggested that miR-146a has the potential to be a biomarker for predicting and diagnosing OA [6]. However, for OA, the expression profile of miRNAs may be different in various tissues.

Recently, a study considered that the level of circulating miR-146a is upregulated in patients with OA [7]. For the cartilage and bone tissues of OA patients, the expression of miR-146a was significantly downregulated [8]. The exosomes of synovial fluid from OA patients also contain miR-146a, which is highly expressed in the early stage and decreases in the later stage of disease $[9,10]$. At present, although many studies have shown that miR$146 \mathrm{a}$ is significantly related to the pathogenesis of OA, its expression level is controversial [11]. Furthermore, its expression profile in various tissues and its diagnostic value for OA still need to be evaluated in detail. This meta-analysis was performed to analyze the expression profile of miR-146a in various tissues of OA patients and evaluate its potential application value as a biomarker.

\section{Methods}

\section{Search strategy}

Two researchers independently searched the PubMed, Embase, Cochrane, Scopes, Reaxys, and Ebscohost databases. These databases were used to obtain all the appropriate studies published up to September 1, 2021. The key words included miR-146a-related items (miR146a, MIR146, miR-146a-3p, miR-146a-5p, MIR146A, MIRN146, MIRN146A, miRNA146A) and OA-related items (osteoarthritis, osteoarthritis, osteoarthrosis). The retrieval strategy adopts the union of the above two types of items. Retrieval does not set language restrictions. Manual retrieval was performed for the references of important reviews to prevent omission.

\section{Inclusion and exclusion criteria}

Two researchers independently screened the items adopted from the search results. The inclusion criteria were as follows: 1 . case-control study; 2 . study comparing the OA population and nonOA healthy population; and 3. study that obtained miR-146a expression profiles, miR-146a-related single nucleotide polymorphisms (SNPs) between two groups, or report diagnostic accuracy of OA with miR-146a. The exclusion criteria were as follows: 1 . repeated reports; 2 . studies on the changes in miR-146a expression before and after OA treatment; 3. studies that did not report miR-146a expression results of a nonOA healthy population; 4. studies comparing OA and rheumatoid arthritis (RA) populations; 5. studies that did not report specific miR-146a expression levels, SNP carrier frequency, or diagnostic accuracy results; and 6, basic research based on animal or cellular levels.
Case reports, editorials, and expert opinions were also excluded.

\section{Data extraction and quality assessment}

Two authors independently extracted data from each eligible study using a predefined data collection sheet, which included the first author's name, publication year, study location, sample size, and test tissue source. Any of following outcomes was recorded: fold-change of miR146a expression between $\mathrm{OA}$ and nonOA health groups by quantitative polymerase chain reaction (qPCR); SNPrelated study reported the carrier frequencies of $\mathrm{OA}$ and control population in each gene phenotype; the sensitivity, specificity and $2 \times 2$ table reported in the diagnostic accuracy study. The results were extracted from figures if specific data were not reported. The QUADAS-2 tool is used to evaluate the quality of eligible studies and consists of four domains: patient selection, index test, reference standard, and flow and timing [12].

\section{Statistical analysis}

The expression profiles, SNP results, and diagnostic accuracy results will be analyzed. The expression profiles are also reported according to different tissue sources. The continuous data were pooled by standardized mean difference (SMD) with its 95\% confidence interval (CI). The binary outcomes were pooled by odds ratio (OR) with its 95\% (CI).The Paule-Mandel estimator is used to estimate the between-study variance $\tau^{\wedge} 2$ [13]. With respect to heterogeneity, the $I^{2}$ statistic was used to estimate the degree of heterogeneity among the studies. When $I^{2} \geq 50 \%$, a random effects model was adopted; otherwise, a fixed effects model was adopted. Publication bias was also evaluated by using funnel plots, Egger's test, and Begg's test. If there was publication bias, the trim and fill method was used to complement the potential study to make the funnel plot symmetrical and to analyze the impact on the pooling results.

We analyzed the diagnostic power of microRNAs in the diagnosis of OA by pooling sensitivity and specificity. Summary receiver operating characteristic (SROC) curves and the area under the curve (AUC) were calculated if feasible [14]. For SNP-related outcomes, the association analyses were performed using the following five genetic models: allelic (W vs. M), dominant (WW + WM vs. MM), recessive (WW vs. WM+MM), heterozygous (WM vs. MM), and homozygous (WW vs. MM). W represents the major wild-type allele, and $M$ represents the minor mutant-type allele [15]. The odds ratios with 95\% CIs were calculated to assess the associations. $\mathrm{R}$ program (version 4.1.0) and RevMan 5.3 were used to perform the analysis. 


\section{Results}

A total of 172 items were identified through databases after removing duplications. After screening the titles and abstracts, 111 items were excluded, and 61 items were obtained to view the full-text article. The following studies were excluded due to: study not report the specific results or results could not be extracted (12); lack of nonOA health control (6); basic research (6); study compare OA and RA population (4); study not related to mir-146a (3); study only report the changes of miR-146a expression before and after OA treatment (3); review (1). Finally, 26 articles were included in the analysis [16-41]. The selection process of eligible study was detailed in the Preferred Reporting Items for Systematic Reviews and Meta-Analysis (PRISMA) flow diagram (Fig. 1). The review was reported according with PRISMA guidelines (Additional file 1: PRISMA Checklist).

The included studies were published from 2010 to 2021 and included two large-scale SNP-related studies [16, 24] and two reported diagnostic accuracy results $[18,27]$. The tissue source for the miR-146a test included plasma, regulatory $\mathrm{T}$ cells, peripheral blood mononuclear cells (PBMCs), local synovial fluid, synovial tissue, synoviocytes, cartilage tissue, and chondrocytes. It should be noted that the people who obtain synovial tissue/cells and cartilage tissue/cells are often those who have experienced joint replacement surgery because of severe OA. Additionally, synoviocytes and chondrocytes have undergone extraction and even culture in vitro. Therefore, these types of cells are analyzed separately from synovial

\section{Identification of studies via databases and registers}

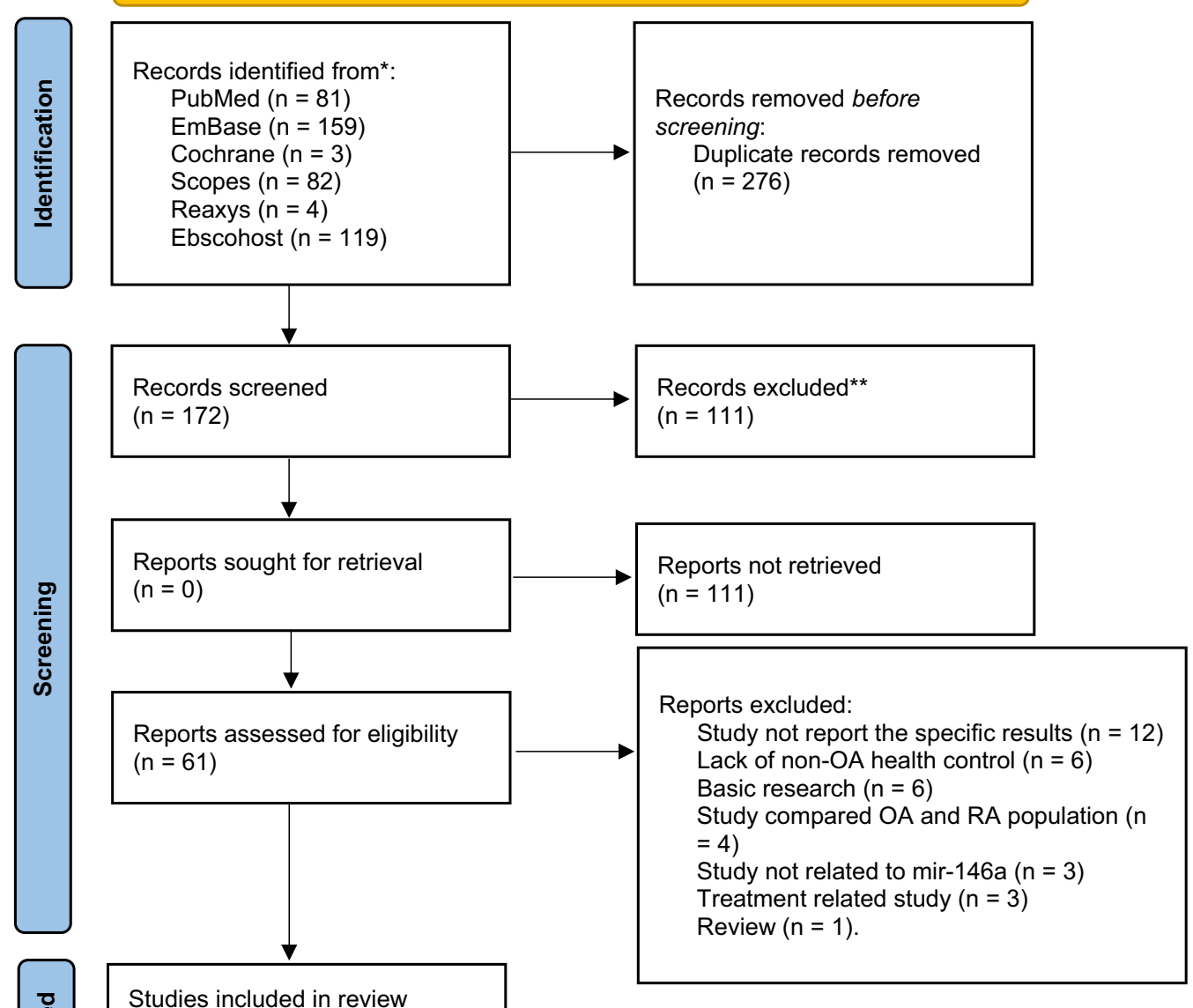

Fig. 1 PRISMA flowchart of the literature search and study selection process 
and cartilage tissue, which are only through the process of tissue separation (Table 1).

For the evaluation of the overall quality, the following factors will affect the risk of bias. Whether the patients were included consecutively or randomly was not described in detail in most studies. Because this metaanalysis is mostly based on case-control studies, the items that "avoid case-control design" are not applicable. In addition, another potential bias was that if the study used joint tissue as the sample source, only people who tended to undergo joint replacement surgery for severe OA were included. However, this study used blood and synovial fluid as sample sources that tended to include a wider range of OA populations. The overall quality of the included studies is shown in Fig. 2.

Seven studies used PBMCs as sample source. A random effects model showed that the expression level of miR-146a in PBMCs was significantly higher in OA patients than in controls (SMD: 1.23; 95\% CI 0.08-2.37; $p=0.035$ ) (Fig. 3A). The results did not detect potential publication bias (Begg's test, $p=0.177$; Egger's test, $p=0.206$ ) (Fig. 4A).

Seven studies used plasma or serum as a sample source. A random effects model showed that the miR-146a expression level was not significantly different between the OA and control groups (SMD: 1.09; 95\% CI -0.06 , 2.24; $p=0.064$ ) (Fig. 3B). Then, publication bias existed (Begg's test, $p=0.453$; Egger's test, $p=0.024$ ). After trim and fill method modification, no significant difference was detected (SMD: $-0.11 ; 95 \% \mathrm{CI}-1.47,1.25$; $p=0.877$ ) (Fig. 4B).

Four studies used cartilage tissue as a sample source. The pooling results based on a random effects model showed that the level of miR-146a in OA cartilage was significantly higher than that in nonOA cartilage (SMD: 6.39; 95\% CI 0.36, 12.4; $p=0.038$ ) (Fig. 3C). Three studies used chondrocytes as a sample source, and a random effect model showed that there was no significant difference between OA patients and controls (SMD: -0.71 ; 95\% CI $-4.15,2.73 ; p=0.687$ ) (Fig. 3D). Whether the

Table 1 Characteristic of included studies

\begin{tabular}{|c|c|c|c|}
\hline Study & Study location & Sample size & Tissue source \\
\hline Miranda-Duarte et al. [16] & Mexican mestizos & 689 & PBMCs (SNP-related study) \\
\hline Nie et al. [17] & China & 132 & Synovial fluid \\
\hline Wu et al. [18] & China & 70 & Plasma \\
\hline Zhang et al. [19] & China & 44 & Cartilage \\
\hline Kmiolek et al. [20] & Poland & 26 & Treg cells \\
\hline Rousseau et al. [21] & France & 133 & Plasma \\
\hline Shao et al. [22] & China & 40 & Cartilage \\
\hline Wang et al. [23] & China & 610 & Plasma \\
\hline Papathanasiou et al. [24] & Greece & 1688 & PBMCs (SNP-related study) \\
\hline Papathanasiou et al. [25] & Greece & 35 & Chondrocytes/synoviocytes \\
\hline Skrzypa et al. [26] & Poland & 30 & Cartilage/serum \\
\hline Ali et al. [27] & India & 28 & Serum \\
\hline Budd et al. [28] & UK & 22 & Chondrocytes \\
\hline Cheleschi et al. [29] & Italy & 10 & Chondrocytes \\
\hline Kopanska et al. [30] & Poland & 31 & Cartilage \\
\hline Soyocak et al. [31] & Turkey & 150 & PBMC \\
\hline Mu et al. [32] & China & 60 & Synovial fluid \\
\hline Zakaria et al. [33] & Egypt & 56 & PBMC \\
\hline Xu et al. [34] & China & 20 & Synovial fluid \\
\hline Wang et al. [35] & China & 14 & PBMC \\
\hline Qian et al. [36] & China & 30 & PBMC \\
\hline Abou-Zeid et al. [37] & Egypt & 105 & PBMC \\
\hline Okuhara et al. [38] & Japan & 72 & PBMC \\
\hline Murata et al. [39] & Japan & 68 & Plasma \\
\hline Niimoto et al. [40] & Japan & 11 & PBMC \\
\hline Nakasa et al. [41] & Japan & 6 & Synovial tissue \\
\hline
\end{tabular}




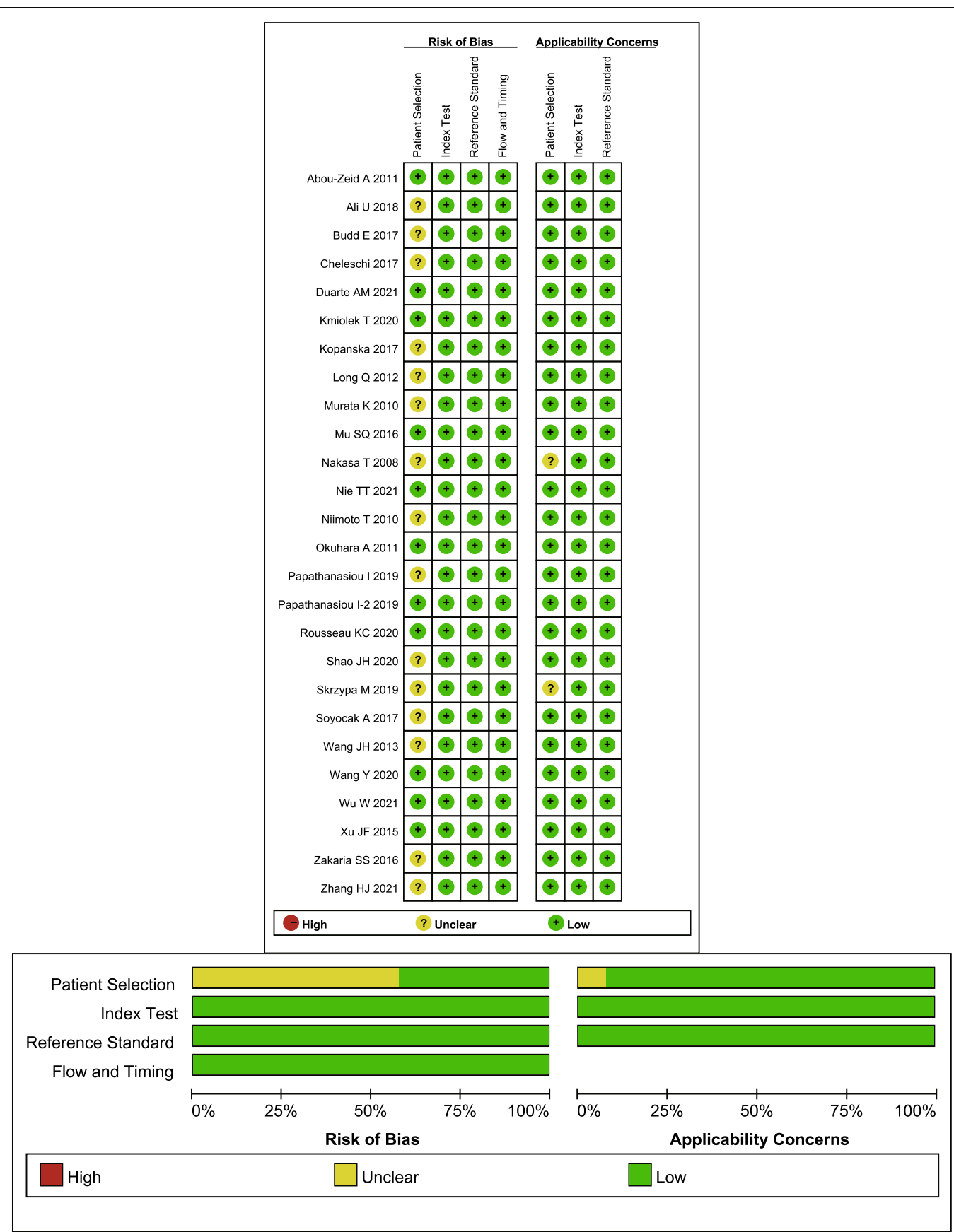

Fig. 2 Risk of bias graph for included primary studies

chondrocyte isolation process impacts the miR-146a level needs further research confirmation.

Three studies researched synovial fluid, but with opposite results $\left(\mathrm{I}^{2}=99 \%, p<0.01\right)$. The pooling results showed that there was no significant difference in miR146a levels between the OA and control groups (SMD: -2.02 ; 95\% CI $-5.60,1.60 ; p=0.27$ ) (Fig. 3E). Other results were based on a single study. These results showed that the miR-146a level was significantly lower in synoviocytes from the OA population than in synoviocytes from the control population (SMD: $-0.97 ; 95 \% \mathrm{CI}$ $-1.68,-0.26 ; p=0.008)$. In synovial tissue and regulatory $\mathrm{T}$ cells, there was no significant difference.

In the PBMC results, subgroup analysis was performed in Kellgren-Lawrence (KL) grade $2-3$ and KL grade 3-4 populations. A random effects model 


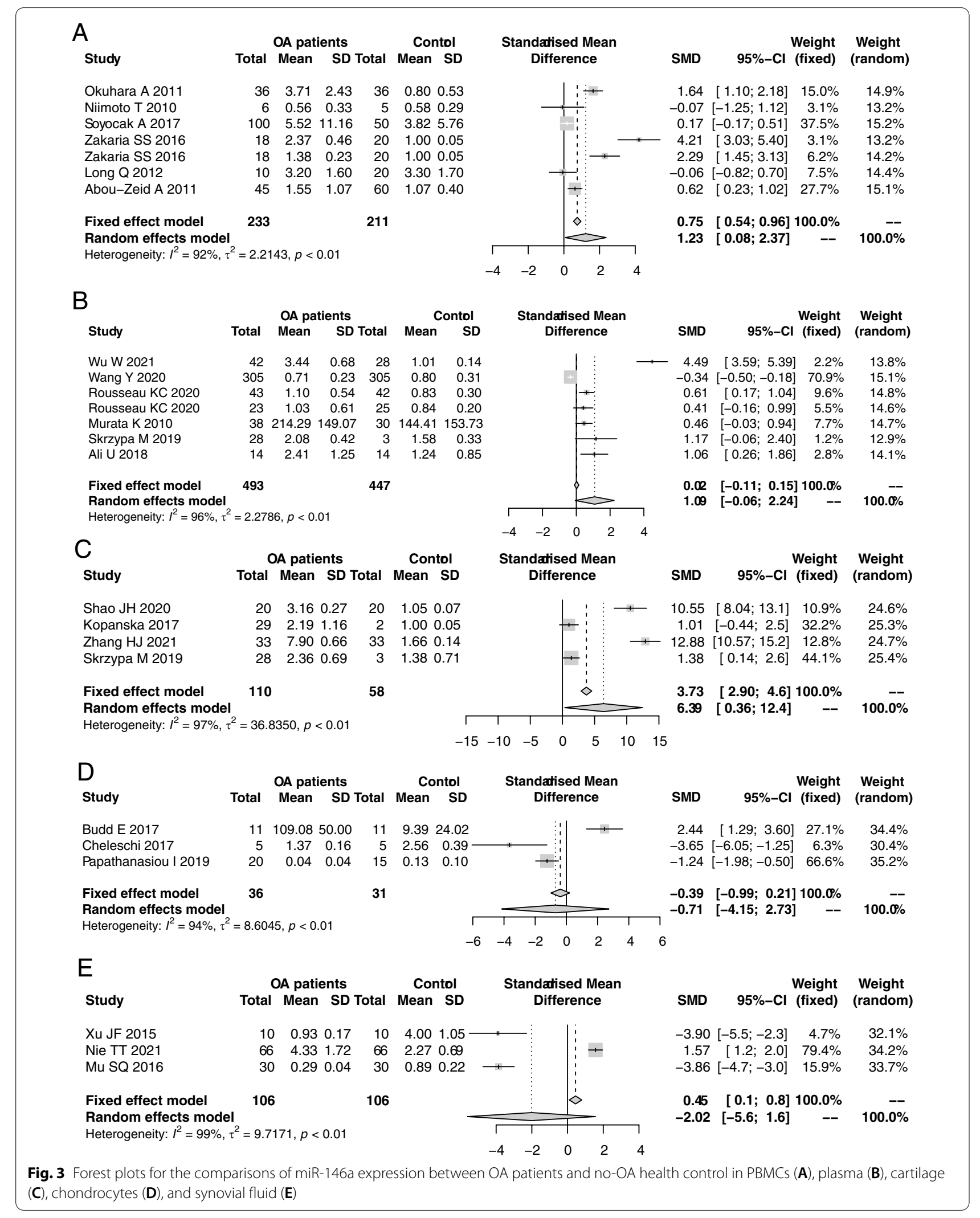



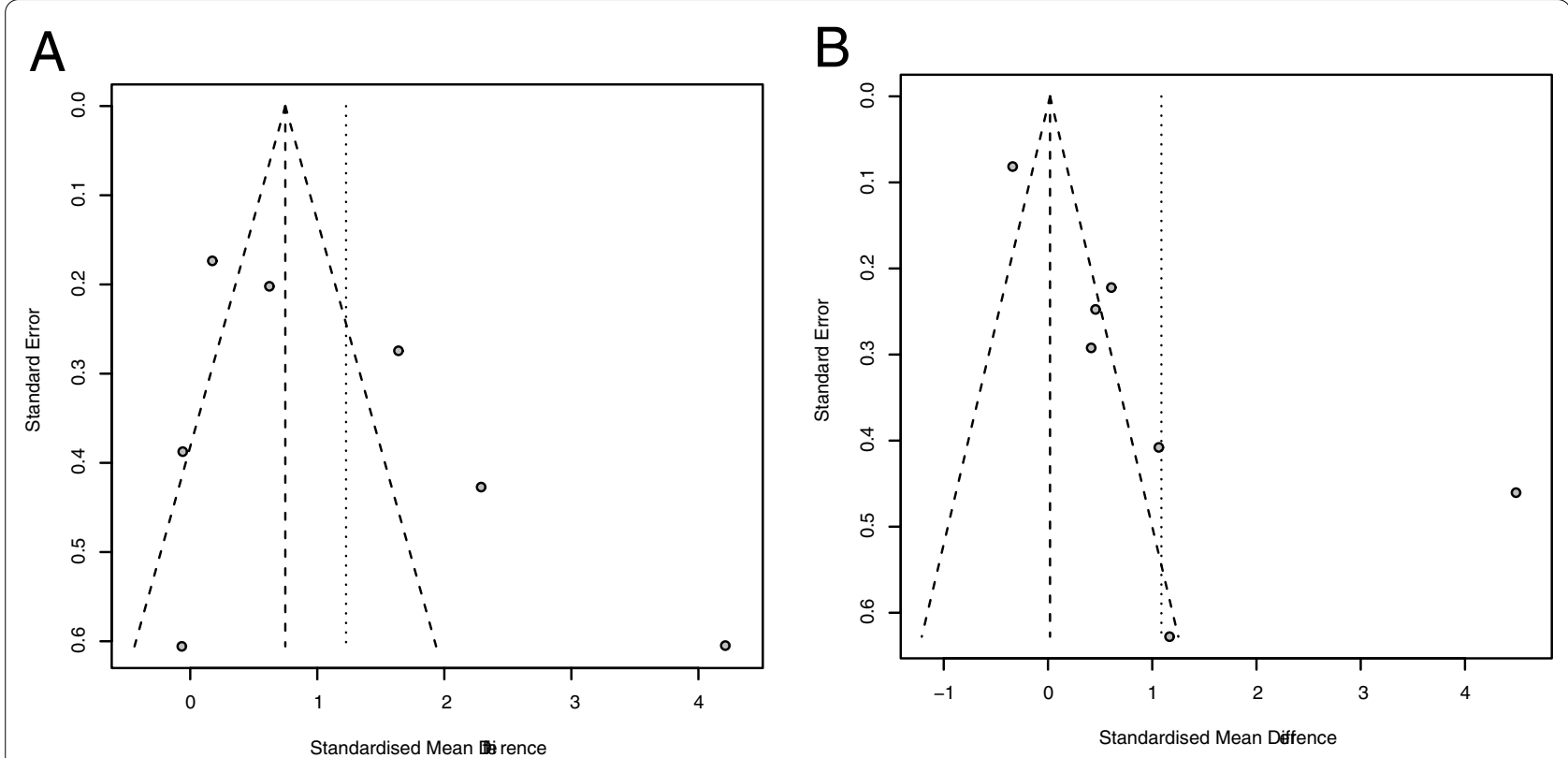

Fig. 4 Funnel plots for the comparisons of miR-146a expression in PBMCs (A) and plasma (B)

showed that miR-146a expression was significantly higher in OA patients than in controls in both the KL grade 2-3 subgroup (SMD: 1.84; 95\% CI 0.37, 3.31; $p=0.014$ ) and the KL grade $3-4$ subgroup (SMD: 1.32; 95\% CI $0.31 ; 2.32 ; p=0.010)$. However, there was no statistical significance between the two subgroups $(p=0.56)$.

Two studies analyzed the diagnostic accuracy of plasma miR-146a for OA $[18,27]$. The pooling sensitivity and specificity results were 0.80 (95\% CI 0.68 , 0.89 ) and 0.93 (95\% CI $0.80,0.98)$, respectively. It is suggested that plasma miR-146a has certain diagnostic ability for OA. However, based on the above results that the plasma miR-146a level was not significantly different between OA patients and controls, the diagnostic accuracy of plasma miR-146a was suspected.

Two studies focused on SNPs of miR-146a [16, 24]. For the rs2910164 locus, there were no significant results in any model [allele model (OR: 1.09; 95\% CI $0.95,1.24 ; p=0.205$ ); heterozygous model (OR: 1.14; 95\% CI 0.96, 1.35; $p=0.125)$; homozygous model (OR: $1.08 ; 95 \%$ CI $0.75,1.56 ; p=0.690)$; dominant model (OR: 1.13; 95\% CI 0.96, 1.34; $p=1.32$ ); recessive model (OR: $1.03 ; 95 \%$ CI 0.72, $1.47 ; p=0.879$ )]. For rs57095329 locus, only one study reported. There were still no significant results [allele model (OR: 1.11; 95\% CI $0.78,1.58 ; p=0.577$ ); recessive model (OR: 1.12 ; $95 \%$ CI $0.77,1.63 ; p=0.554)]$.

\section{Discussion}

This meta-analysis first analyzed the difference in miR-146a expression between OA and nonOA control patients in various tissue samples. The results showed that the expression level of miR-146a in PBMCs was significantly higher in $\mathrm{OA}$ patients than in nonOA healthy people. However, there was no significant difference in plasma. The expression level of miR-146a in cartilage tissue was significantly higher in OA patients than in controls. However, no significant difference was found in chondrocytes. Based on a single study, the expression of miR-146a in synoviocytes was significantly lower in OA patients than in controls (Fig. 5).

In addition, although in the KL 2-3 and KL 3-4 subgroups, the miR-146a in PBMCs was significantly higher in OA patients than in controls. There was no significant difference between the two subgroups. Therefore, it cannot be supported that OA KL grade has a significant effect on the expression of miR-146a. Then, although the results of the diagnostic accuracy analysis suggested that plasma miR-146a can help in the diagnosis of $\mathrm{OA}$, there was no significant difference in miR-146a levels between OA patients and controls. Therefore, the diagnostic accuracy of plasma miR-146a needs to be further determined. The rs2910164 and rs57095329 loci of miR-146a also did not show that the mutation would increase the risk of OA. 


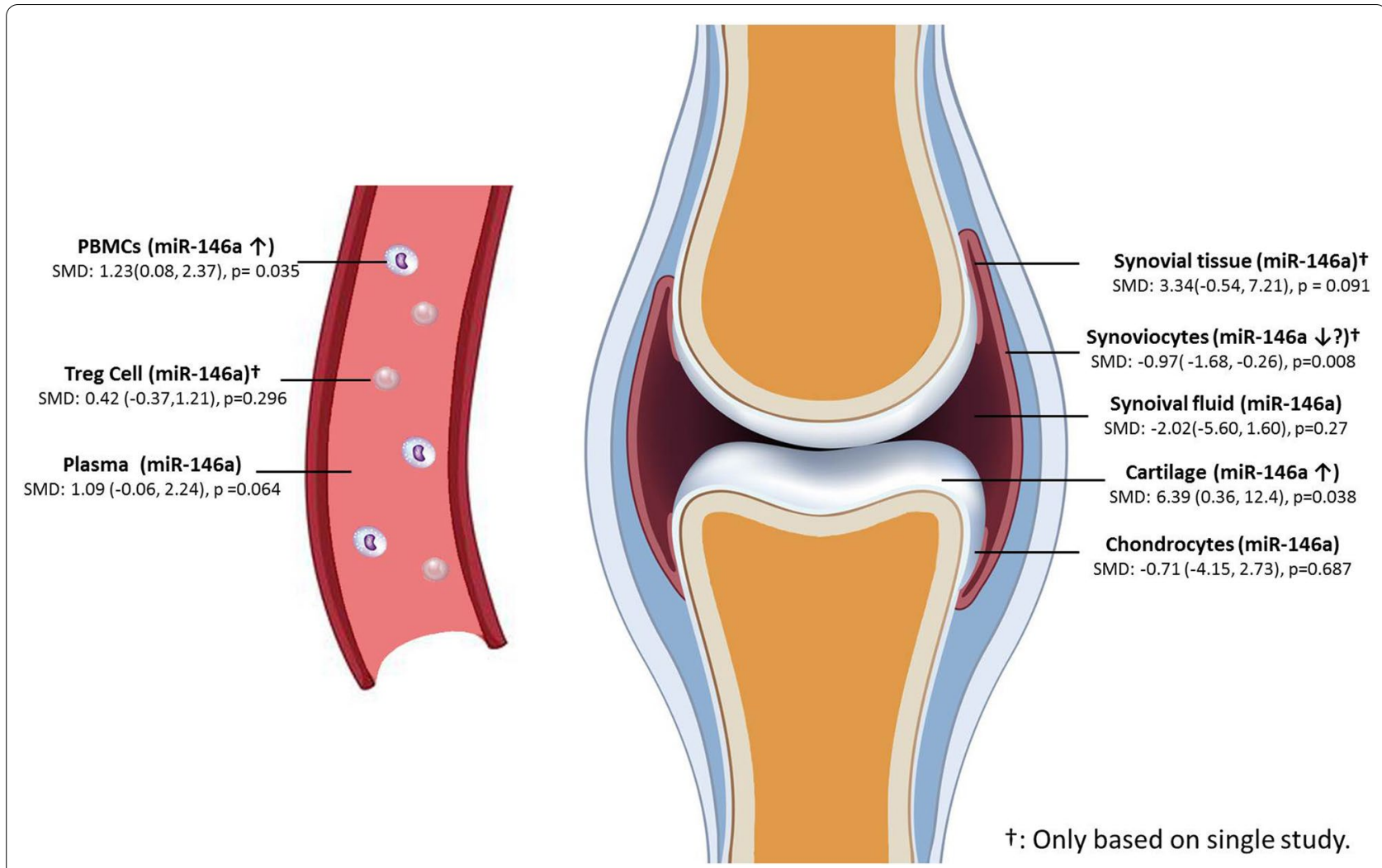

Fig. 5 Schematic diagrams illustrating miR-146a expression in different tissue origin of OA patients

The key target genes of miR-146a-5p (cumulative weighted context score $\leq-0.4$ ) included IRAK1 and TRAF6, which are more closely related to the pathogenesis of OA (TargetScan database: http://www.targe tscan.org). However, the miR-146a-3p-targeting genes have less evidence to correlate with $\mathrm{OA}$ at present. Although the miR-146a SNP-related study did not support that the mutation could increase the OA risk, it suggested that the mutation reduced the expression level of miR-146a and then increased the expression of IRAK1 and TRAF6. Therefore, it confirmed the targeted regulation of miR-146a-5p on IRAK1 and TRAF6 [24].

However, the basic research results of miR-146a and its targeting genes impact OA are still controversial. Studies have suggested that upregulation of miR-146a-5p or inhibition of IRAK1 can alleviate inflammation [42], cartilage degradation [43], and autophagy [44]. Inhibiting TRAF6 expression also alleviated the inflammatory response, reduced the degradation of extracellular matrix [45], and alleviated chondrocyte apoptosis [46]. In addition, in PBMCs, miR-146a-5p also inhibits inflammation through the regulation of IRAK1 [47]. In regulatory T cells, miR146a-5p exerts immune regulation ability by inhibiting the NF-kb signaling pathway [48]. However, one study showed that miR-146a-5p can promote chondrocyte apoptosis [22].

At present, most viewpoints still believe that miR146a-5p exerts its inflammatory inhibitory effect by targeting TRAF6 and IRAK1 to inhibit the NF-kb pathway. Therefore, the upregulation of miR-146a mainly plays a protective role in OA development. This might explain the high miR-146a expression level in the early stage of $\mathrm{OA}$ and the low level in the late stage [49]. Because the NF-kb pathway also controls miR-146a expression [50], another possible explanation is that miR-146a-5p is a negative feedback molecule of the NF-kb pathway. Therefore, the upregulated expression of miR-146a indicates a higher level of inflammation. Combined with the results of this study, it is suggested that the high expression of miR-146a in PMBCs and cartilage tissue represents a more active NF-kb signaling pathway in patients with OA. A further increase in miR-146a expression may be a means to inhibit the level of inflammation.

\section{Advantages and limitations}

The advantage of this study is that it first evaluated the expression profile of miR-146a in different tissues of OA patients by meta-analysis. This study still has the following limitations. First, this work was performed at the 
study level instead of at the individual level. Second, data acquisition is mainly obtained from bar charts or scatter charts that might not be accurate enough. Third, there is obvious heterogeneity in the results that might lead to a no robust conclusion. Fourth, the number of studies from some tissue sources is still small, making the results vulnerable to publication bias and tending to be positive.

\begin{abstract}
Abbreviations
OA: Osteoarthritis; PBMCs: Peripheral blood mononuclear cells; SMD: Standardized mean difference; SNPs: Single nucleotide polymorphisms; RA: Rheumatoid arthritis; qPCR: Quantitative polymerase chain reaction; Cl: Confidence interval; SROC: Summary receiver operating characteristic; AUC: Area under the curve; KL: Kellgren-Lawrence; OR: Odds ratio.
\end{abstract}

\section{Supplementary Information}

The online version contains supplementary material available at https://doi. org/10.1186/s13018-022-02989-7.

Additional file 1: PRISMA 2020 checklist items for meta-analysis and systematic review.

\section{Acknowledgements}

None.

\section{Authors' contributions}

Study concepts and design: C-MF; Literature research: J-NL; Data acquisition and analysis: J-NL; Statistical analysis: SL; Manuscript preparation: J-NL and C-MF; Manuscript editing: J-NL and C-MF; Manuscript review: J-NL and C-MF. All authors read and approved the final manuscript.

\section{Funding}

None.

\section{Availability of data and materials}

All data generated or analyzed during this study are included in this published article.

\section{Declarations}

Ethics approval and consent to participate

Not applicable.

\section{Consent for publication}

Not applicable.

\section{Competing interests}

The authors declare that they have no competing interests.

Received: 19 November 2021 Accepted: 3 February 2022

Published online: 05 March 2022

\section{References}

1. Katz JN, Arant KR, Loeser RF. Diagnosis and treatment of hip and knee osteoarthritis: a review. JAMA. 2021;325:568-78.

2. Hunter DJ, March L, Chew M. Osteoarthritis in 2020 and beyond: a Lancet Commission. Lancet. 2020;396:1711-2.

3. Oliviero A, Della Porta G, Peretti GM, Maffulli N. MicroRNA in osteoarthritis: physiopathology, diagnosis and therapeutic challenge. Br Med Bull. 2019;130:137-47.

4. Malemud CJ. MicroRNAs and osteoarthritis. Cells. 2018;7:92.
5. Hesari R, Keshvarinia M, Kabiri M, et al. Comparative impact of platelet rich plasma and transforming growth factor-beta on chondrogenic differentiation of human adipose derived stem cells. Bioimpacts. 2020;10:37-43.

6. Kriegsmann M, Randau TM, Gravius S, et al. Expression of miR-146a, miR155 , and miR-223 in formalin-fixed paraffin-embedded synovial tissues of patients with rheumatoid arthritis and osteoarthritis. Virchows Arch. 2016;469:93-100.

7. Jones TL, Esa MS, Li KHC, et al. Osteoporosis, fracture, osteoarthritis and sarcopenia: a systematic review of circulating microRNA association. Bone. 2021;152:116068.

8. Nugent M. MicroRNAs: Exploring new horizons in osteoarthritis. Osteoarthritis Cartilage. 2016;24:573-80.

9. Dole NS, Delany AM. MicroRNA variants as genetic determinants of bone mass. Bone. 2016;84:57-68.

10. Liu DH, Jheng YC, Chen PY, et al. Frontier review of the roles of exosomes in osteoarthritis. J Chin Med Assoc. 2021;84:754-6.

11. Cong L, Zhu Y, Tu G. A bioinformatic analysis of microRNAs role in osteoarthritis. Osteoarthritis Cartilage. 2017;25:1362-71.

12. Whiting PF, Rutjes AW, Westwood ME, et al. QUADAS-2: a revised tool for the quality assessment of diagnostic accuracy studies. Ann Intern Med. 2011;155:529-36.

13. Langan D, Higgins JPT, Simmonds M. Comparative performance of heterogeneity variance estimators in meta-analysis: a review of simulation studies. Res Synth Methods. 2017;8:181-98.

14. Leeflang MM. Systematic reviews and meta-analyses of diagnostic test accuracy. Clin Microbiol Infect. 2014:20:105-13.

15. Alidoust M, Hamzehzadeh L, Rivandi M, et al. Polymorphisms in noncoding RNAs and risk of colorectal cancer: a systematic review and metaanalysis. Crit Rev Oncol Hematol. 2018;132:100-10.

16. Miranda-Duarte A, Borgonio-Cuadra VM, Gonzalez-Huerta NC, et al. Are functional variants of the microRNA-146a gene associated with primary knee OA? Evidence in Mexican mestizo population. Mol Biol Rep. 2021:48:1549-57.

17. Nie T, Zhang C, Zhang G, et al. LncRNA CALML3-AS1 regulates chondrocyte apoptosis by acting as a sponge for miR-146a. Autoimmunity. 2021;54:336-42.

18. Wu W, Xuan Y, Ge Y, et al. Plasma miR-146a and miR-365 expression and inflammatory factors in patients with osteoarthritis. Malays J Pathol. 2021;43:311-7.

19. Zhang H, Zheng W, Li D, et al. miR-146a-5p promotes chondrocyte apoptosis and inhibits autophagy of osteoarthritis by targeting NUMB. Cartilage. 2021;2021:19476035211023550.

20. KmiolekT, Rzeszotarska E, Wajda A, et al. The interplay between transcriptional factors and MicroRNAs as an important factor for Th17/Treg balance in RA patients. Int J Mol Sci. 2020;21:7169.

21. Rousseau JC, Millet M, Croset M, et al. Association of circulating microRNAs with prevalent and incident knee osteoarthritis in women: the OFELY study. Arthritis Res Ther. 2020;22:2

22. Shao J, Ding Z, Peng J, et al. MiR-146a-5p promotes IL-1 beta-induced chondrocyte apoptosis through the TRAF6-mediated NF-kB pathway. Inflamm Res. 2020;69:619-30.

23. Wang Y, LiY, Jia D, et al. Correlation between single nucleotide polymorphisms in CXCR4 microRNA binding site and the susceptibility to knee osteoarthritis in Han Chinese population. J Clin Lab Anal. 2021;35:e23600.

24. Papathanasiou I, Mourmoura E, Balis C, et al. Impact of miR-SNP rs2910164 on miR-146a expression in osteoarthritic chondrocytes. Adv Med Sci. 2020;65:78-85.

25. Papathanasiou I, Trachana V, Mourmoura E, et al. DNA methylation regulates miR-140-5p and miR-146a expression in osteoarthritis. Life Sci. 2019;228:274-84

26. Skrzypa M, Szala D, Gablo N, et al. miRNA-146a-5p is upregulated in serum and cartilage samples of patients with osteoarthritis. Pol Przegl Chir. 2019:91:1-5.

27. Ali U, Lakkireddy C, Ahmed SI, et al. Quantitative epigenetic analysis reveals significance of lipid metabolism regulatory miRNA-33a in defining different categories of arthritis. Meta Gene. 2019;19:160-7.

28. Budd E, de Andres MC, Sanchez-Elsner T, et al. MiR-146b is down-regulated during the chondrogenic differentiation of human bone marrow derived skeletal stem cells and up-regulated in osteoarthritis. Sci Rep. 2017;7:46704. 
29. Cheleschi S, De Palma A, Pecorelli A, et al. Hydrostatic pressure regulates microRNA expression levels in osteoarthritic chondrocyte cultures via the Wnt/beta-catenin pathway. Int J Mol Sci. 2017;18:133.

30. Kopanska M, Szala D, Czech J, et al. MiRNA expression in the cartilage of patients with osteoarthritis. J Orthop Surg Res. 2017;12:51.

31. Soyocak A, Kurt H, Ozgen M, et al. miRNA-146a, miRNA-155 and JNK expression levels in peripheral blood mononuclear cells according to grade of knee osteoarthritis. Gene. 2017;627:207-11.

32. Mu SQ, Sun S, Wang Y, et al. Altered expression levels of miRNAs in joint fluid of knee osteoarthritis patients. Shandong Med J. 2016;56:5-7.

33. Zakaria SS, Gaballah HH, El Saadany HM. Micro RNA-146a expression, NF-kB/P65 activity and serum pentosidine levels as potential biomarkers for disease severity in primary knee osteoarthritis patients. Egypt Rheumatol. 2016:38:319-25.

34. Xu JF, Zhang SJ, Zhao C, et al. Altered microRNA expression profile in synovial fluid from patients with knee osteoarthritis with treatment of hyaluronic acid. Mol Diagn Ther. 2015;19:299-308.

35. Wang JH, Shih KS, Wu YW, et al. Histone deacetylase inhibitors increase microRNA-146a expression and enhance negative regulation of interleukin-1 beta signaling in osteoarthritis fibroblast-like synoviocytes. Osteoarthr Cartil. 2013;21:1987-96.

36. Qian L, Gao DW, Wang GS, et al. Relationship between the single nucleotide polymorphisims in pre-miR-146a rs2910164 and expression of miR-146a in rheumatoid arthritis. Chin J Microbiol Immunol (China). 2012;32:253-7.

37. Abou-Zeid A, Saad M, Soliman E. MicroRNA 146a expression in rheumatoid arthritis: association with tumor necrosis factor-alpha and disease activity. Genet Test Mol Biomarkers. 2011;15:807-12.

38. Okuhara A, Nakasa T, Shibuya $H$, et al. Changes in microRNA expression in peripheral mononuclear cells according to the progression of osteoarthritis. Mod Rheumatol. 2012;22:446-57.

39. Murata K, Yoshitomi H, Tanida S, et al. Plasma and synovial fluid microRNAs as potential biomarkers of rheumatoid arthritis and osteoarthritis. Arthritis Res Ther. 2010;12:R86

40. Niimoto T, Nakasa T, Ishikawa M, et al. MicroRNA-146a expresses in interleukin-17 producing T cells in rheumatoid arthritis patients. BMC Musculoskelet Disord. 2010;11:209.

41. Nakasa T, Miyaki S, Okubo A, et al. Expression of microRNA-146 in rheumatoid arthritis synovial tissue. Arthritis Rheum. 2008;58:1284-92.

42. Avenoso A, D'Ascola A, Scuruchi M, et al. miR146a up-regulation is involved in small HA oligosaccharides-induced pro-inflammatory response in human chondrocytes. Biochim Biophys Acta Gen Subj. 2021;1865:129731.

43. Li W, Wang Y, Tang Y, et al. Quercetin alleviates osteoarthritis progression in rats by suppressing inflammation and apoptosis via inhibition of IRAK1/NLRP3 signaling. J Inflamm Res. 2021;14:3393-403.

44. Chen G, Gao X, Wang J, et al. Hypoxia-induced microRNA-146a represses Bcl-2 through Traf6/IRAK1 but not Smad4 to promote chondrocyte autophagy. Biol Chem. 2017;398:499-507.

45. Chen X, Yu M, Xu W, et al. Rutin inhibited the advanced glycation end products-stimulated inflammatory response and extra-cellular matrix degeneration via targeting TRAF- 6 and BCL-2 proteins in mouse model of osteoarthritis. Aging (Albany NY). 2021;13:22134-47.

46. Jiang J, Zhang J, Wu C, et al. Knockdown of TRAF6 inhibits chondrocytes apoptosis and inflammation by suppressing the NF-kappaB pathway in lumbar facet joint osteoarthritis. Mol Cell Biochem. 2021;476:1929-38.

47. Venuti A, Musarra-Pizzo M, Pennisi R, et al. HSV-1 \EGFP stimulates miR146a expression in a NF-kappaB-dependent manner in monocytic THP-1 cells. Sci Rep. 2019;9:5157

48. Wang J, Zhai X, Guo J, et al. Long non-coding RNA DQ786243 modulates the induction and function of CD4(+) Treg cells through Foxp3-miR146a-NF-kappaB axis: implications for alleviating oral lichen planus. Int Immunopharmacol. 2019;75:105761.

49. Yamasaki K, Nakasa T, Miyaki S, et al. Expression of MicroRNA-146a in osteoarthritis cartilage. Arthritis Rheum. 2009;60:1035-41.

50. Mossallam GI, Fattah RA, Mahmoud HK. Nuclear factor-kappaB1 and microRNA-146a polymorphisms and risk of acute graft versus host disease post allogeneic stem cell transplantation. Immunobiology. 2020;225:151876

\section{Publisher's Note}

Springer Nature remains neutral with regard to jurisdictional claims in published maps and institutional affiliations.
Ready to submit your research? Choose BMC and benefit from:

- fast, convenient online submission

- thorough peer review by experienced researchers in your field

- rapid publication on acceptance

- support for research data, including large and complex data types

- gold Open Access which fosters wider collaboration and increased citations

- maximum visibility for your research: over $100 \mathrm{M}$ website views per year

At BMC, research is always in progress.

Learn more biomedcentral.com/submissions 